\title{
Catálogo de Apoidea da Região Neotropical (Hymenoptera, Colletidae). V. Xeromelissinae ${ }^{1}$
}

\author{
Jesus Santiago Moure ${ }^{2}$ \\ Danúncia Urban ${ }^{2}$
}

\begin{abstract}
Catalogue of the Apoidea of the Neotropical Region (Hymenoptera, Colletidae) V. Xeromelissinae. The type localities, depositary museums, taxonomical comments and geographical occurrences of the Neotropical Xeromelissinae are given. Flowers visited and nesting habits of some species are presented. The following new combinations are proposed: Chilicola cupheae (Schrottky, 1905) and Chilicola (Anoediscelis) dalmeidai (Moure, 1946). Chilicola friesella is the new name given to Chilicola friesei Herbst, 1920, the homonym resulted by the publication of Oediscelis friesei Ducke, 1907 as Chilicola (Oediscelis) friesei by Michener, 1995.

KEY WORDS. Hymenoptera, Colletidae, Xeromelissinae, Neotropical
\end{abstract}

Xeromelissinae são abelhas delicadas que lembram os Hylaeinae pelo corpo alongado, com numerosas espécies chilenas e que ocorrem desde o México até o extremo sul da América. A subfamília foi proposta por COCKERELl (1926) ao descrever a fêmea de Xeromelissa wilmattae Cockerell, 1926 com palpos maxilares e labiais peculiares, os labiais tri-articulados, sendo o primeiro artículo mais longo que os dois restantes, e os maxilares enormes sobrepassando a gálea e quase tão largos como a mesma. Michener (1944) incluiu Chilicola Spinola, 1851, Oediscelis Philippi, 1866 e Idioprosopis Meade-Waldo, 1914 em Chilicolinae, comentando que Xeromelissa Cockerell, 1926 provavelmente também pertenceria a esta subfamília. MOURE (1946) considerou Chilicolinae e Xeromelissinae como subfamílias distintas, citou os três gêneros relacionados por MICHENER (1944), acrescentando Pseudiscelis Friese, 1906 e um gênero novo Oediscelisca. TORO \& MICHENER (1975) consideraram Chilicolinae, nome proposto por MICHENER (1944) como sinônimo de Xeromelissinae e incluiram Hylaeosoma Ashmead, 1898 como subgênero de Chilicola deixando Oediscelis e Oediscelisca como subgêneros ou sinônimos de Chilicola.

TORO \& MOLDENKE (1979), revisando os Xeromelissinae chilenos, relacionaram quatro gêneros: Xeromelissa, com uma única espécie do Peru; Xenochilicola gen. nov., Chilimelissa gen. nov. e Chilicola, este último com os subgêneros: Chilicola $\mathbf{s}$. str., Idioprosopis, Oediscelis e os subgêneros novos Anoediscelis, Chilioediscelis, Heteroediscelis e Stenoediscelis. MichENER (1995) dividiu os Xeromelissinae em Chilicolini com dois gêneros Chilicola e Xenochilicola e Xeromelissini incluindo Xeromelissa e Chilimelissa. Considerou válidos os seguintes subgêneros de Chilicola: Chilicola s. str., Chilioediscelis, Hylaeosoma, Prosopoides, Pseudiscelis, Anoediscelis e Oediscelis; colocou o subgênero Stenoediscelis na sinonímia Anoediscelis e,

1) Contribuição número 1322 do Departamento de Zoologia, Universidade Federal do Paraná.

2) Departamento de Zoologia, Universidade Federal do Paraná. Caixa Postal 19020, 81531-990 Curitiba, Paraná, Brasil. Bolsista do CNPq. 
Idioprosopis, Oediscelisca e Heteroediscelis como sinônimos de Oediscelis. MichENER \& ROZEN (1999) descreveram mais um gênero, Geodiscelis, com caracteres das duas tribos e sugeriram o uso dos gêneros sem a classificação tribal.

Neste catálogo são mantidas as sinonímias propostas por MICHENER (1995) e confirmadas por MICHENER (2000). As espécies de Chilicola não relacionadas em subgênero pelos autores permanecem sem esta indicação. Os demais procedimentos utilizados neste trabalho são as abreviações latinas comuns em taxonomia, as citações mais frequentes estão relacionadas nas "Referências Bibliográficas". Novas informações sobre ocorrências, visitas a flores e comportamento de nidificação foram obtidas na literatura.

Acrônimos: (AMNH) American Museum of Natural History, New York, Estados Unidos; (BMNH) The Natural History Museum, Londres, Inglaterra; (CAS) California Academy of Sciences, San Francisco, Estados Unidos; (CTV) Coleção Toro, Valparaíso, Chile; (DZUP) Departamento de Zoologia da Universidade Federal do Paraná, Coleção Pe. J.S. Moure, Curitiba, Brasil; (MIZT) Museo ed Istituto di Zoologia Sistematica, Universitá di Torino, Itália; (MHNS) Museo Nacional de Historia Natural, Santiago, Chile; (MPEG) Museu Paraense Emílio Goeldi, Belém, Brasil; (MNHP) Muséum National d'Histoire Naturelle, Paris, França; (MNHU) Museum für Naturkunde der Humboldt-Universität zu Berlin, Alemanha; (MZSP) Museu de Zoologia, Universidade de São Paulo, São Paulo, Brasil; (NHMV) Naturhistorisches Museum, Viena, Austria; (SEMK) Snow Entomological Museum, University of Kansas, Lawrence, Estados Unidos; (UCV) Universidad Catolica de Valparaíso, Chile; (USDA) Bee Biology and Systematics Laboratory, Logan, Utah, Estados Unidos; (USNM) National Museum of Natural History, Washington, Estados Unidos.

\section{CONTEÚDO}

\begin{tabular}{|c|c|}
\hline & \\
\hline Chilicola ......................... & \\
\hline Chilicola (Anoediscelis). & $c$ \\
\hline …................ 7 & Geoc \\
\hline .......... 8 & Xenochilicola.. \\
\hline hilicola $(H$ & Xeromelissa.... \\
\hline hilicola (Oediscelis) ..........................11 & Índice ........................ \\
\hline
\end{tabular}

\section{Chilicola Spinola, 1851}

Chilicola Spinola, 1851, in Gay. Hist. Fis. Pol. Chile, Zool., 6: 210. Espécie-tipo: Chilicola rubriventris Spinola, 1851. Designação de Sandhouse, 1943. Proc. U. S. Natl. Mus. 92: 537.

aequatoriensis Benoist. EQUADOR, [Pichincha], Quito (localidade-tipo).

Chilicola aequatoriensis Benoist, 1942. Ann. Soc. Ent. France 111: 81. Holótipo macho coletado em 27.X.1930. Depositado no MNHP.

Biologia. Benoist, 1942: 81 (em uma haste de Rubus L.). 
cupheae (Schrottky). PARAGUAI, Villa Encarnación (localidade-tipo). comb. nov. Scrapteroides cupheae Schrottky,1905. An. Ci. Paraguayos 1(4): 2. Um cótipo no MZSP.

Oediscelis cupheae; Ducke, 1912. Zool. Jahrb. Syst. 34: 83.

Taxonomia. Schrottky, 1905: 2 (descreveu a espécie no gênero Scrapteroides Gribodo, 1894, sob reserva, considerando-o um Panurgidae [= Panurginae, Andrenidae]). - Ducke, 1912: 83 (referiu-se a um cótipo no MZSP, sem indicar o sexo do exemplar).

Biologia. Schrottky, 1905: 3 (em flores de Cuphea mesostemon Kóehne em novembro, e em flores de Raphanus radiola DC. em fevereiro).

longitarsa Spinola. CHILE, Províncias do sul (localidade-tipo) e Valdivia.

Chilicola longitarsa Spinola, 1851, in Gay. Hist. Fis. Pol. Chile, Zool., 6:

212. Síntipo fêmea no MIZT.

quitensis Benoist. EQUADOR, [Pichincha], Quito (localidade-tipo), Rumipamba, Cotollao, estrada de Calderon, Palmira, Valle de Lloa, Páramos de Alogo, Otavalo.

Chilicola quitensis Benoist, 1942. Ann. Soc. Ent. France 111: 79. Síntipos fêmea e macho depositados no MNHP.

Taxonomia. Benoist, 1942: 81 (próxima de C. styliventris (= Chilicola (Anoediscelis) styliventris (Friese, 1908)).

Biologia. Benoist, 1942: 80-81 (observou exemplares em Otavalo nos meses de janeiro, fevereiro, maio, outubro e dezembro e, nas demais localidades durante o ano todo; descreveu ninhos encontrados em um galho de sarça [Rubus fruticosus L.], em forma de galeria escavada na qual estavam dispostas as células em série linear, cada uma forrada por uma fina película transparente feita pela fêmea antes do aprovisionamento).

\section{Chilicola (Anoediscelis) Toro \& Moldenke, 1979}

Chilicola (Anoediscelis) Toro \& Moldenke, 1979. An. Mus. Hist. Nat. 12: 131. Espécie-tipo: Oediscelis herbsti Friese, 1906. Designação original. - Michener, 1992 [1994]. Folia Entomol. Mex. 85: 89; syn.: Chilicola (Stenoediscelis).

Chilicola (Stenoediscelis) Toro \& Moldenke, 1979: 135. Espécie-tipo: Oediscelis inermis Friese, 1906. Designação original.

Taxonomia. Toro \& Moldenke, 1979: 131 (chave para as espécies do subgênero Anoediscelis); 135 (chave para as espécies do subgênero Stenoediscelis). Michener, 1992 [1994]: 89 (comentou estar usando Stenoediscelis num sentido amplo para incluir três espécies que TORO \& MOLDENKE (1979) colocaram no subgênero Heteroediscelis: C. olmue Toro \& Moldenke, 1979, C. orophila Toro \& Moldenke, 1979 e C. minor (Philippi, 1866)). - Michener, 1995: 337 (repetiu a posição das três espécies colocadas por TORO \& MOLDENKE (1979) em Heteroediscelis, não só pelos caracteres subgenéricos mas pelo sétimo esterno do macho).

ashmeadi (Crawford). CosTA RICA, S. José (localidade-tipo de H. ashmeadi e de $O$. costaricensis), San Ignacio de Acosta; Cartago, Tres Rios, Cartago e Turrialba; Puntarenas, Monteverde (1.300-1.400m). MÉXICO, Oaxaca (loca- 
lidade-tipo de $P$. howardiella); $3 \mathrm{~km}$ ao norte de Cuyoyepeji; Chiapas, $14 \mathrm{~km}$ ao sul de Ixtapa; 6,4 km ao sul de Simojovel; Comitán; Sumidero; Puebla, Tehuacán; Michoacán, Uruapan; Guerrero, $11 \mathrm{~km}$ a leste de Chilpancingo $(1.540 \mathrm{~m}) ;$ Jalisco, Guadalajara, $16 \mathrm{~km}$ a sudoeste de Ciudad Guzmán (2.000m); Nayarit, Islas Tres Marías, Maria Cleofas. Guatemala, Antigua (1.500-1.600m). El Salvador, Mt. San Salvador, Santa Tecla (900-950m). Hylaeosoma ashmeadi Crawford, 1906. Tr. Amer. Ent. Soc. 32: 161. Síntipo fêmea no USNM, $n^{\circ} 10.074$. Examinado por J.S. Moure.

Oediscelis costaricensis Friese, 1916. Stett. ent. Ztg. 77: 302. Síntipos: oito fêmeas e dois machos; destes, um síntipo macho no USNM, examinado por J.S. Moure e um no AMNH, citado por Michener 1992 [1994]; os demais provavelmente no MNHU.

Prosopis howardiella Cockerell, 1918. Ann. Mag. Nat. Hist. (9) 12: 423. Síntipo macho no USNM.

Hylaeosoma ashmeadi; Cockerell, 1919. Proc. U. S. Natl. Mus. 55: 185. Hylaeus howardiellus; Meade-Waldo, 1923. Gen. Insectorum 181: 32.

Chilicola ashmeadi; Éickwort, 1967. Jour. Kans. Ent. Soc. 40 (1): 42; syn.: Oediscelis costaricensis. - Snelling, 1982. Bull. So. California Acad. Sci. 81 (1): 5; syn.: Hylaeus howardiellus.

Chilicola (Anoediscelis) ashmeadi; Michener, 1992 [1994]. Folia Entomol. Mex. 85: 91.

Taxonomia. Eickwort, 1967. Jour. Kansas Ent. Soc. 40: 43 (localidades na Costa Rica). - Michener, 1992 [1994]: 91, figs 1, 2, 5, 6, 13-15 (citou um síntipo de $O$. costaricensis no AMNH e novas ocorrências).

Biologia. Eickwort, 1967: 42-73, figs 1-26 (descreveu ninhos de C. ashmeadi encontrados em galhos mortos e ocos de ramos caidos de Erythrina poeppigiana (Skeels), alguns deles usados também por Stigmus (Gonostigmus) temporalis [= Stigmus temporalis Kohl, 1892]. As células dos ninhos dispostas em série linear, eram forradas com uma secreção semelhante a celofane e com provisão semi-líquida; das células basais emergiram fêmeas e das apicais os machos. As fêmeas coletavam pólen somente em Solanaceae: Saracha procumbens (Cav.) Ruiz \& Pav., Solanum nigrum L.,, Solanum umbellatum Mill. e Solanum wendlandii Hook. Para obtenção de néctar, visitavam flores de Polymnia maculata Cav., Bidens pilosa L., Melampodium divaricatum (Rich.) DC., Jaegeria hirta Less. e Galinsoga Ruiz \& Pav. Comparou a larva com a de Hylaeus Fabricius, 1793 e Euryglossa Smith, 1853). - Linsley \& Cazier, 1970. Jour. Kansas Ent. Soc. 43 (3): 258 (referiram-se ao trabalho de Eickwort, 1967 que observou esta abelha na Costa Rica).

herbsti (Friese). CHILE, Rancagua (localidade-tipo); Valparaíso, La Dormida; Coquimbo, Río Laguna (3000m), Santiago.

Oediscelis herbsti Friese, 1906. Ztschr. Hym. Dipt. 6: 227. Holótipo fêmea provavelmente no MNHU.

Chilicola herbsti; Herbst, 1920. Deutsch. ent. Ztschr.: 199.

Chilicola (Anoediscelis) herbsti; Toro \& Moldenke, 1979: 131. 
Biologia. Herbst, 1922. Ent. Mitt. 9 (2): 64 (sobre flores de Achillaea [sic] millefolium L. [=Achillea millefolium], Anthemis cotula Blanco., Tanacetum parthenifolium Sch. Bip., Sedum L., Salvia splendens Ker Gawl., Antirrhinum majus L. e Azara celastrina D. Don.).

Morfologia. Toro \& Moldenke, 1979: 175, figs 184-192 (caracteres do macho e da fêmea). - Aravena \& Toro, 1985. Rev. Chil. Ent. 12: 179, figs 4, 17, 22 (ferrão e estruturas associadas).

inermis (Friese). CHILE, Concepción (localidade-tipo), Corral; Atacama, Tierra Amarilla, Las Juntas; Coquimbo, Río Laguna, Illapel; Valparaíso, Malleco, Lonquimay. ArgentinA, Mendoza, Chacras de Coria.

Oediscelis inermis Friese, 1906. Flora og Fauna 10: 7. Síntipos: três machos coletados em 18.XII.1904 em Concepción e uma fêmea coletada em março, em Corral. Um síntipo macho com o n ${ }^{0} 13.207$ no USNM, demais síntipos provavelmente no MNHU.

Chilicola inermis; Claude-Joseph, 1926. Ann. Sci. Nat., Zool., Paris, 10: 116

Chilicola (Stenoediscelis) inermis; Toro \& Moldenke, 1979: 135.

Chilicola (Anoediscelis) inermis; Michener, 1995: 336.

Taxonomia. Joergensen, 1912. Zool. Jahrb. Syst. 32: 92 (ocorrência na Argentina). - Ruiz, 1936. Rev. Chilena Hist. Nat. 40: 165 (ocorrência em Coquimbo).

Biologia. Joergensen, 1912: 92 (sobre flores de Larrea divaricata Cav.). Herbst, 1922. Ent. Mitt. 9 (2): 64 (Hypochaeris [sic] lessingii Reiche [= Hypochoeris lessingii], Baccheris [sic] rosmarinifolia Hook.. \& Arn. [= Baccharis rosmarinifolia], Eryngium paniculatum Cav. \& Dombey ex Delar., Phacelia circinata Jacq. F., Azara integrifolia, Cristaria intermedia Gay, Leuceria eriochloena [sic] Remy [= Leuceria eriochlaena], Achillaea [sic] millefolium L. [= Achillea millefolium], Anthemis cotula Blanco., Matthiola annua Sweet, Antirrhinum majus L., Lobelia salicifolia Sweet, em dezembro em grande número sobre Gymnophytum [sic] polycephalum Clos. [= Gymnophyton polycephalum]). - Claude-Joseph, 1926.: 116-119; figs 1, 1 a 4 (Observou ninhos em pequenos tubos de bambu com diâmetro aproximado de $2 \mathrm{~mm}$, onde a fêmea secretou uma substância viscosa e construiu uma célula cilíndrica com pouco mais de $5 \mathrm{~mm}$ de altura, fechada no fundo e com abertura superior; depois a fêmea visitou flores recolhendo nectar e pólen de Stachys albicaulis Lindl., Podanthus mitiqui Lindl., Taraxacum dens-leonis Desf.[= Taraxacum officinale Weber], Brassica campestris L., Tecoma Juss., Lychnis L. e Salvia L. Após a postura fechou a célula com uma membrana que passou a servir de fundo para a célula seguinte).

Morfologia. Toro \& Moldenke, 1979: 175, figs 209 a 217 (caracteres do macho e da fêmea). - Aravena \& Toro, 1985. Rev. Chil. Ent. 12: 179, figs 4, 15, 28 (ferrão e estruturas associadas). - Toro \& Magunacelaya, 1987. Acta Ent. Chilena 14: 15, figs 7 a 10 (músculos femurais). 
mailen Toro \& Moldenke. CHILE, Antofagasta, Toconao (localidade-tipo), ChiuChiu.

Chilicola (Stenoediscelis) mailen Toro \& Moldenke, 1979. An. Mus. Hist. Nat. 12: 137. Holótipo macho e alótipo na CTV. Parátipos: 8 fêmeas e 370 machos, depositados no SEMK, AMNH, MHNS, UCV e CTV.

Taxonomia. Toro \& Moldenke, 1979: 137 (compararam com C. inermis Friese, 1906).

Morfologia. Toro \& Moldenke, 1979: 175, figs 218 a 226 (caracteres do macho e da fêmea).

minor (Philippi). CHILE, Aconcagua, Laillai (localidade-tipo); Concepción (localidade-tipo de O. albida); Valparaíso; Talca, Las Mercedes; Malleco, Icalma.

Oediscelis minor Philippi, 1866. Stett. ent. Ztg. 27: 110. Material tipo não examinado, provavelmente depositado no MHNS - Toro \& Moldenke, 1979: 128; syn.: Oediscelis albida.

Oediscelis albida Friese, 1906. Ztschr. Hym. Dipt. 6: 228. Holótipo fêmea, coletado em 28. II por Herbst, provavelmente no MNHU.

Chilicola albida; Herbst, 1920. Deutsch. ent. Ztschr.: 200.

Ordiscelis[sic] albida; Toro \& Moldenke, 1979: 128.

Chilicola (Heteroediscelis) minor; Toro \& Moldenke, 1979: 128.

Chilicola (Anoediscelis) minor; Michener, 1995: 337.

Taxonomia. Friese, 1906: 227 (redescrição, ocorrência de O. minor). - Toro \& Moldenke, 1979: 129 (variação quanto ao tamanho, manchas amarelas cefálicas e processo espiniforme dos trocanteres posteriores dos machos, e pontuação e alargamento da cabeça das fêmeas).

Biologia. Herbst, 1922. Ent. Mitt. 9 (2): 63 a 68 (em flores de Cristaria intermedia Gay, Gymnophytum [sic] polycephalum Clos. [= Gymnophyton polycephalum] e Echinocactus ceratites [sic] Otto ex Pfeiff [= Echinocactus ceratistes]. Comentou sua predileção por Compostas [Asteraceae]; e as observou, muito numerosas, como um enxame de mosquitos, sobre flores de Gymnophytum [sic] polycephalum. Encontrou-as em galerias abandonadas por besouros, galhos secos e ocos de bambus [Poaceae], bambu da India e Bambusa Schref. e machos voando nas proximidades, no solo e nas flores. Citou como C. albida, em flores de Colletia ferox Gill \& Hook., Phacelia circinata Jacq. F., Azara integrifolia Ruiz \& Pav., Armeria maritima (Miller) Willd., Achillea millefolium L., Anthemis cotula Blanco., Tanacetum parthenifolium Sch. Bip., Baccheris [sic] confertifolia Colla [= Baccharis confertifolia], Baccheris [sic] rosmarinifolia Hook. \& Arn.[= Baccharis rosmarinifolia], Santolina chamaecyparissus L., Helianthus tuberosus L., Loasa tricolor Lindl. Weinm., Salvia splendens Ker Gawl., Aquilejia [sic] vulgaris L. [= Aquilegia vulgaris], Cheiranthus cheiri L., Sedum L.). - Toro \& Moldenke, 1979: 129 (em flores de Escalonia [sic] pulverulenta Pers. [= Escallonia pulverulenta]).

Morfologia. Toro \& Moldenke, 1979: 173, figs 159 a 167 (caracteres do macho e da fêmea). 
plebeia Spinola. ChILE, Províncias do sul (localidade-tipo), Coquimbo, La Serena, Los Choros, Vicuña, Talinay, El Tofo; Valparaíso, La Dormida, Viña del Mar (localidade-tipo de C. friesei); Osorno.

Chilicola plebeia Spinola, 1851, in Gay. Hist. Fis. Pol. Chile, Zool., 6: 211. Síntipo fêmea no MIZT. - Toro \& Moldenke, 1979: 133; syn.: Chilicola friese $i$ Herbst.

Chilicola plebeja[sic]; Cockerell, 1919. Proc. U. S. Natl. Mus. 55: 186.

Chilicola friesei Herbst, 1920. Deutsch. ent. Ztschr.: 201; nom. praeoc. Síntipos fêmea e macho. Depositário desconhecido.

Chilicola (Anoediscelis) plebeia; Toro \& Moldenke, 1979: 133.

Chilicola friesella nom. nov. para Chilicola friesei Herbst, 1920 non Oediscelis friesei Ducke, 1907 que foi publicada por MicHENER (1995: 337) como Chilicola (Oediscelis) friesei, resultando na homonímia.

Biologia. Herbst, 1922. Ent. Mitt. 9 (2): 63 (obervou C. plebeia sobre flores de Senecio adenotrichius DC., Baccheris [sic] rosmarinifolia Hook. \& Arn. [= Baccharis rosmarinifolia] e Senecio rutaceus Phil.); 64 (como C. friesei sobre flores de Achillaea [sic] millefolium [= Aquillea millefolium], Anthemis cotula Blanco., Sedum L., Lippia citriodora H. B. K., Lobelia polyphylla Fr. Meigen, Azara celastrina D. Don., Loasa tricolor Lindl. Weinm., Phrygilanthus aphyllus Eichl.). - Claude-Joseph, 1926. Ann. Sci. Nat., Zool., Paris, 10: 119; fig. 3, 1 a 3 (observou C. friesei usando galerias abandonadas de Ceratina Latreille, 1802, após limpar as galerias as fêmeas construíram suas células e fecharam a abertura com um tampão membranoso; publicou a figura de um velho ninho de Manuelia Vachal, 1905, com uma abertura circular que foi fechada pela Chilicola).

Morfologia. Toro \& Moldenke, 1979: 175, figs 193 a 201 (caracteres do macho e da fêmea).

styliventris (Friese). PERU (localidade-tipo), Tingo [Maria].

Oediscelis styliventris Friese, 1908. Flora og Fauna 10: 9. Síntipos: dois machos e duas fêmeas provavelmente no MNHU.

Chilicola (Anoediscelis) styliventris; Michener, 1995: 336.

Taxonomia. Cockerell, 1926. Ann. Mag. Nat. Hist. (9) 17: 218 (ocorrência).

yali Toro \& Moldenke. CHILE, Valparaíso, La Dormida (localidade-tipo).

Chilicola (Anoediscelis) yali Toro \& Moldenke, 1979. An. Mus. Hist. Nat.

12: 134. Holótipo na CTV. Cinco parátipos machos depositados no SEMK, AMNH, MHNS, UCV e CTV.

Taxonomia. Toro \& Moldenke, 1979: 134, figs 202 a 208 (compararam com C. herbsti (Friese, 1906); caracteres do macho).

\section{Chilicola (Chilicola) Spinola, 1851}

Chilicola Spinola, 1851. Espécie-tipo: Chilicola rubriventris Spinola, 1851. Designação de Sandhouse, 1943. Proc. U. S. Natl. Mus. 92: 537.

Taxonomia. Michener, 1995: 338 (comparou Chilicola s. str. com os subgêneros Chilioediscelis e Oediscelis). 
aisenensis Toro \& Moldenke. CHILE, Aisén (localidade-tipo).

Chilicola (Chilicola) aisenensis Toro \& Moldenke, 1979. An. Mus. Hist.

Nat. 12: 102. Holótipo macho na CTV.

Morfologia. Toro \& Moldenke, 1979: 169, figs 19 a 25 (caracteres do macho).

colliguay Toro \& Moldenke. CHILE, Valparaíso (localidade-tipo).

Chilicola (Chilicola) colliguay Toro \& Moldenke, 1979. An. Mus. Hist.

Nat. 12: 100. Holótipo macho e alótipo na CTV. Parátipos, cinco

machos e oito fêmeas, depositados no AMNH, MHNS, UCV e CTV.

Chilicola colliquey[sic]; Michener, 1995: 338.

Chilicola (Chilicola) colliquay [sic]; Michener, 1995: 339.

Morfologia. Toro \& Moldenke, 1979: 169, figs 10 a 18 (caracteres do macho e da fêmea).

pangue Toro \& Moldenke. CHILE, Coquimbo, El Pangue (localidade-tipo).

Chilicola (Chilicola) pangue Toro \& Moldenke, 1979. An. Mus. Hist. Nat.

12: 103. Holótipo na CTV.

Morfologia. Toro \& Moldenke, 1979: 169, figs 26 a 32 (caracteres do macho).

rubriventris Spinola. CHILE, Províncias do sul (localidade-tipo); Atacama, La Junta; Coquimbo, El Pangue; Santiago, Farellones.

Chilicola rubriventris Spinola, 1851, in Gay. Hist. Fis. Pol. Chile, Zool., 6:

211. Síntipo fêmea no MIZT.

Biologia. Toro \& Moldenke, 1979: 100 (coletados sobre Adesmia melanthes Phil.).

Morfologia. Toro \& Moldenke, 1979: 169, figs 1 a 9 (caracteres do macho e da fêmea). - Aravena \& Toro, 1985. Rev. Chil. Ent. 12: 177, figs 1, 11, 21 (ferrão e hemitergitos associados). - Toro \& Magunacelaya, 1987. Acta Ent. Chilena 14: 14-15, figs 1 a 4 (músculos femurais).

\section{Chilicola (Chilioediscelis) Toro \& Moldenke, 1979}

Chilicola (Chilioediscelis) Toro \& Moldenke, 1979. An. Mus. Hist. Nat. 12: 104. Espécie-tipo: Chilicola andina Toro \& Moldenke, 1979. Designação original.

Taxonomia. Toro \& Moldenke, 1979: 104 (chave para as espécies).

andina Toro \& Moldenke. CHILE, Coquimbo, norte da Laguna Dam (localidadetipo) 8.000 pés, Maños [sic]del Toro [= Baños del Toro], Curamahuida.

Chilicola (Chilioediscelis) andina Toro \& Moldenke, 1979. An. Mus. Hist.

Nat. 12: 105. Holótipo macho na CAS, alótipo e um parátipo macho na CTV.

Morfologia. Toro \& Moldenke, 1979: 171, figs 33 a 41 (caracteres do macho e da fêmea). 
araucana Toro \& Moldenke. Argentina, Río Negro, San Carlos de Bariloche (localidade-tipo).

Chilicola (Chilioediscelis) araucana Toro \& Moldenke, 1979. An. Mus. Hist. Nat. 12: 108. Holótipo macho na CTV.

Morfologia. Toro \& Moldenke, 1979: 171, figs 51 a 58 (caracteres do macho).

patagonica Toro \& Moldenke. ARgentinA, Santa Cruz, Los Antiguos (localidaele-tipo).

Chilicola (Chilioediscelis) patagonica Toro \& Moldenke, 1979. An. Mus.

Hist. Nat. 12: 106. Holótipo macho, alótipo, um parátipo macho e uma fêmea na CTV.

Morfologia. Toro \& Moldenke, 1979: 171, figs 42 a 50 (caracteres do macho e da fêmea). - Aravena \& Toro, 1985. Rev. Chil. Ent. 12: 179, figs 7, 24, 27 (ferrão e estruturas associadas).

\section{Chilicola (Hylaeosoma) Ashmead, 1898}

Hylaeosoma Ashmead, 1898. Psyche 8: 284. H. longiceps citada pelo autor como "Type" mas não descrita. Primeira espécie incluída Hylaeosoma longiceps Ashmead, 1900. Espécie-tipo por monotipia subsequente.

Hyloeosoma [sic] Ashmead, 1899. Psyche 9: 376.

Chilicola (Hylaeosoma); Toro \& Michener, 1975. Jour. Kansas Ent. Soc. 48 (3): 352.

Taxonomia. Ashmead, 1898: 284 (considerou Hylaeosoma próximo de Dasypoda Latreille, 1802 e de Calliopsis Smith, 1853; como espécie-tipo citou $H$. longiceps Ashmead, MS.; não a descreveu). - Toro \& Michener, 1975: 352 (redescreveram o subgênero).

gracilis Michener \& Poinar. REPÚBLICA DOMINICANA (localidade-tipo) (em âmbar dominicano, provavelmente proveniente das minas da Cordilheira Setentrional entre Santiago e Puerto Plata).

Chilicola (Hylaeosoma) gracilis Michener \& Poinar, 1996. Jour. Kansas

Ent. Soc. 69(4) suppl.: 358. Holótipo macho na Coleção Poinar, em

âmbar, University of California, Berkeley; fêmea no AMNH.

Taxonomia. Michener \& Poinar, 1996: 358 (comentaram que a fêmea poderia ou não ser da mesma espécie que o macho).

griswoldi Michener. MÉxICO, Michoacán, Huetano (localidade-tipo); México, km 135, rodovia 55, Puente Calderón, ao norte de Ixtapan de la Sal.

Chilicola (Hylaeosoma) griswoldi Michener, 1992 [1994]. Folia Entomol.

Mex. 85: 85. Holótipo macho no USDA. Parátipos: duas fêmeas no

USDA e uma fêmea no Dept. of Entomology, Cornell University, Ithaca,

New York.

Taxonomia. Michener, 1992 [1994]: 84, figs 7 a 9 (comparou com Chilicola mexicana Toro \& Michener, 1975).

Biologia. Michener, 1992 [1994]: 85 (sobre flores de Tripogandra amplexicaulis (Klotz ex B. Clarke) Woodson). 
huberi (Ducke). BRASIL, Ceará, Serra de Baturité (localidade-tipo) 600-800m.

Oe. [sic] Huberi[sic] Ducke, 1908. Rev. d'Ent., Caen, 27: 63. Lectótipo fêmea no MPEG.

Chilicola (Hylaeosoma) huberi; Michener, 1995: 340.

Taxonomia. Ducke, 1908: 63 (comparou com Oediscelis prosopoides [= Chilicola (Prosopoides) prosopoides (Ducke, 1907)]). - Brooks \& Michener, 1999. Jour. Hym. Res. 8 (2); 137 (designaram o lectótipo fêmea que foi encontrado no SEMK a ser retornado ao MPEG).

Biologia. Ducke, 1908: 63 (sobre flores de Ruellia L.).

longiceps (Ashmead). WeSt INDIES [PEQUENAS ANTILHAS], St. Vincent, Kingstown (localidade-tipo). MÉxICO, Jalisco, Chamela.

Hylaeosoma longiceps Ashmead, 1898. Psyche 8: 284; nom. nudum.

Hylaeosoma longiceps Ashmead, 1900. Trans. Ent. Soc. Lond. II: 215.

Síntipos: uma fêmea e um macho no USNM; um fêmea no BMNH sob o número 17. A. 1809; examinados por J.S. Moure.

Chilicola (Hylaeosoma) longiceps; Michener, 1995: 340.

Taxonomia. Michener, 1992 [1994]. Folia Entomol. Mex. 85: 86 (comparou C. longiceps com Chilicola griswoldi Michener, 1992 [1994] e Chillicola polita Michener, 1992 [1994], citou ocorrência no México).

megalostigma (Ducke). BRASIL, Ceará, Serra de Baturité (localidade-tipo) 700m. Oe.[sic] megalostigma Ducke, 1908. Rev. d'Ent., Caen, 27: 62. Lectótipo [sem indicação do sexo] 31.VII.1908 designado por Nascimento, 1979 e lectoalótipo coletado em 29.VII.1908 depositados no MPEG. Lectoparátipos no MPEG, SEMK, BMNH e presumivelmente também no DZUP e MZSP.

Chilicola (Hylaeosoma) megalostigma; Michener, 1995: 339.

Taxonomia. Ducke, 1908: 62 (comparou com Oediscelis prosopoides [= Chilicola (Prosopoides) prosopoides (Ducke, 1907)]). - Brooks \& Michener, 1999. Jour. Hym. Res. 8 (2): 133 (informações sobre o material-tipo, caracteres morfológicos).

Biologia. Ducke, 1908: 63 (em flores de Borreria verticillata G. F. W. Mey.).

mexicana Toro \& Michener. MÉxICO, México, $25 \mathrm{~km}$ lesde de Toluca (localidadetipo) (2.900m); México, Río Frio $3.005 \mathrm{~m}$; Cortez Pass (2.745m); $32 \mathrm{~km}$ a leste de Toluca (2.714m); Morelos, Yautepec (1.130m); Hidalgo, El Chico.

Chilicola (Hylaeosoma) mexicana Toro \& Michener, 1975. Jour. Kansas Ent. Soc. 48: 353. Holótipo macho, alótipo fêmea e 15 parátipos machos e 87 fêmeas, coletados no México por expedições da University of Kansas. Holótipo e parátipos no SEMK. Parátipos na Escuela Nacional de Agricultura, Chapingo, MÉXICO; CTV, BMNH, DZUP, USNM e $\mathrm{AMNH}$.

Taxonomia. Michener, 1992 [1994]. Folia Entomol. Mex. 85: 85 (redescrição e ocorrência). 
polita Michener. MéXICO, Veracruz, Xico, Texolo Falls [Quedas] (1.400m) (localidade-tipo); Chiapas, Pk. Lago Belgica; Puebla, 22 km N de Xicotepec de Juarez, 1.070m; Tamaulipas, Rancho del Cielo próximo de Gomez Farias $1.000 \mathrm{~m}$; Oaxaca, Metate, $85,5 \mathrm{~km}$ SW de Tuxtepec (900m). Guatemala, Zacapa, $3,5 \mathrm{~km}$ SE de La Union, $1.500 \mathrm{~m}$. PANAMÁ, Chiriquí, Potrerillos. Costa Rica, San José, San Antonio de Escazú.

Chilicola (Hylaeosoma) polita Michener, 1992 [1994]. Folia Entomol. Mex. 85: 87. Holótipo fêmea no SEMK. Parátipos: macho e fêmea no Amer. Ent. Inst., Gainesville, Florida, USA; um macho no USDA.

Taxonomia. Michener, 1992 [1994]: 87, figs 3, 4, 10 a 12 (comparou com Chilicola megalostigma (Ducke, 1908)). - Brooks \& Michener, 1999. Jour. Hym. Res. 8 (2): 134 (distribuição geográfica, notas morfológicas); 137-138 (novas ocorrências).

Biologia. Brooks \& Michener, 1999: 134 (sobre Cuphea P. Br.).

stenocephala Brooks \& Michener. COLÔMBIA, Amazonas, La Chorrera (localidade-tipo).

Chilicola (Hylaeosoma) stenocephala Brooks \& Michener, 1999. Jour. Hym. Res. 8 (2): 134. Holótipo macho e dois parátipos, um macho e uma fêmea, no BMNH; um parátipo macho no SEMK.

Taxonomia. Brooks \& Michener, 1999: 133; figs 5 a 14 (chave para $C$. stenocephala, C. megalostigma e C. polita; figuras do holótipo, alótipo e caracteres morfológicos).

\section{Chilicola (Oediscelis) Philippi, 1866}

Oediscelis Philippi, 1866. Stett. ent. Ztg. 27: 109. Espécie-tipo: Oediscelis vernalis Philippi, 1866. Designação de Cockerell, 1919. Proc. U. S. Natl. Mus. 55: 185.

Idioprosopis Meade-Waldo, 1914. Ann. Mag. Nat. Hist. (8)14: 451. Espécie-tipo: Idioprosopis chalcidiformis Meade-Waldo, 1914. Monotípico e designação original. - Michener, 1995: 337; syn.: Chilicola (Oediscelis).

Oediscelisca Moure, 1946: 243. Espécie-tipo: Oediscelis friesei Ducke, 1907. Designação original. Michener, 1995: 337; syn.: Chilicola (Oediscelis).

Chilicola (Heteroesdiscelis)[sic] [= Chilicola (Heteroediscelis)] Toro \& Moldenke, 1979: 112. Espécie-tipo: Chilicola mantagua Toro \& Moldenke, 1979: 114. Designação original. - Michener, 1995: 337; syn.: Chilicola (Oediscelis).

Taxonomia. Toro \& Moldenke, 1979: 109 (colocaram como espécie-tipo, por engano, Oediscelis plebeia [sic] Philippi, em vez de Oediscelis vernalis Philippi, 1866 e elaboraram chave para espécies de Chilicola (Oediscelis); 112 - 114 (descrição do subgênero e chave pra as espécies de $C$. (Heteroediscelis); 139 (chave para as espécies de C. (Idioprosopis)). - Michener, 1995: 337 (comentou o laps. de Toro \& Moldenke na descrição do subgênero ao relacionar Oediscelis plebeia como espécie-tipo).

chalcidiformis (Meade-Waldo). ARgENTINA, Patagonia, Chubut, Valley [sic] de Lago Blanco (localidade-tipo). CHILE, Malleco, Lonquimay; Magallanes, Tres Brazos, Lago Toro, Mina Rica. 
Idioprosopis chalcidiformis Meade-Waldo, 1914. Ann. Mag. Nat. Hist.

(8)14: 451. Holótipo macho depositado no BMNH.

Chilicola (Idioprosopis) chalcidiformis; Toro \& Moldenke, 1979: 139.

Taxonomia. Cockerell, 1926. Ann. Mag. Nat. Hist. (9) 17: 218 (examinou o tipo no BMNH).

Morfologia. Toro \& Moldenke, 1979: 175, figs 227 a 235 (caracteres do macho e da fêmea). - Aravena \& Toro, 1985: 178 figs 3, 14, 23 (ferrão e estruturas associadas). - Toro \& Magunacelaya, 1987. Acta Ent. Chilena 14: 15, figs 11 a 14 (músculos femurais).

dalmeidai (Moure). BRASIL, Paraná, Curitiba (localidade-tipo). comb. nov. Oediscelisca dalmeidai Moure, 1946. Livro de Homenagem F.F. d'Almeida 27: 246. Holótipo macho no DZUP.

Biologia. Moure, 1946: 247 (voando juntamente com Oediscelisca friesei [= Chilicola (Oediscelis) friesei (Ducke, 1907)]).

Taxonomia. Michener, 1995: 337 (incluiu Oediscelisca na sinonímia de Chilicola (Oediscelis) e citou somente a espécie-tipo O. friesei Ducke, 1907).

deserticola Toro \& Moldenke. CHILE, Atacama, Pueblo Hundido (localidade-tipo). Chilicola (Heteroediscelis) deserticola Toro \& Moldenke, 1979. An. Mus.

Hist. Nat. 12: 121. Holótipo macho e um parátipo macho na CTV.

Biologia. Toro \& Moldenke, 1979: 122 (sobre Heliotropium stenophyllum Hook. \& Arn.).

Morfologia. Toro \& Moldenke, 1979: 173, figs 120 a 126 (caracteres do macho).

diaguita Toro \& Moldenke, 1979. CHILE, Coquimbo (localidade-tipo).

Chilicola (Heteroediscelis) diaguita Toro \& Moldenke, 1979. An. Mus.

Hist. Nat. 12: 116. Holótipo macho e alótipo na CTV.

Morfologia. Toro \& Moldenke, 1979: 171, figs 86 a 92 (caracteres do macho).

erithropoda Toro \& Moldenke. CHILE, Atacama, Pueblo Hundido (localidade-tipo). Chilicola (Heteroediscelis) erithropoda Toro \& Moldenke, 1979. An. Mus.

Hist. Nat. 12: 127. Holótipo macho na CTV.

Biologia. Toro \& Moldenke, 1979: 127 (coletado sobre Heliotropium stenophilum [sic] [= Heliotropium stenophyllum $]$ ).

Morfologia. Toro \& Moldenke, 1979: 173, figs 152 a 158 (caracteres do macho).

friesei (Ducke). BRAsIL, Minas Gerais, Barbacena (localidade-tipo); Paraná, Curitiba $900 \mathrm{~m}$.

Oediscelis Friesei [sic] Ducke, 1907. Ztschr. Hym. Dipt. 7: 364. Holótipo macho. Depositário desconhecido. Oediscelisca friesei; Moure, 1946: 244. 
Ooediscelis [sic] friesei; Moure, 1946: 245.

Chilicola (Oediscelis) friesei; Michener, 1995: 337.

Taxonomia. Ducke, 1907: 364 (próxima a Oediscelis vernalis Philippi, 1866). - Moure, 1946: 245, figs 1 e 2 (descreveu a fêmea e redescreveu o macho).

Biologia. Moure, 1946: 246 (geralmente em flores de cenoura (Daucus carota L.) e Drymaria Willd. ex Schult.).

gutierrezi Moure. CHILE, Valdivia, Panguipulli (localidade-tipo); Cautín, Sierra de Nahuelbuta; Osorno, oeste de Purranque.

Chilicola gutierrezi Moure, 1947. Mus. Paranaense Publ. Avuls. 3: 3. Holótipo macho no DZUP.

Chilicola (Oediscelis) gutierrezi; Michener, 1995: 337.

Taxonomia. Moure, 1947: 4 (comparou-a com Chilicola plebeia Spinola, 1851).

Morfologia. Toro \& Moldenke, 1979: 177, figs 261 a 266 (caracteres do macho).

hahni Herbst. CHILE, Valparaíso (localidade-tipo).

Chilicola Hahni [sic] Herbst, 1923. Rev. Chilena Hist. Nat. 27: 73. Síntipos fêmeas e machos. Um síntipo fêmea na CAS, examinado por J.S. Moure. Chilicola (Oediscelis) hahni; Michener, 1995: 337.

Taxonomia. Herbst, 1923: 73 (achou-a semelhante a C. plebeia Spinola, 1851 e comentou ter depositado tipos em sua coleção). - Toro \& Moldenke, 1979: 143 (não incluíram a espécie em subgênero).

Morfologia. Toro \& Moldenke, 1979: 177, figs 252 a 260 (caracteres do macho e da fêmea).

Biologia. Herbst, 1923: 74 (sobre flores de Phacelia circinata Jacq. F.).

lonco Toro \& Moldenke, 1979. ChILE, Valparaíso, Belloto (localidade-tipo); Santiago, El Canelo; Nuble, El Roble; Concepción, Collico; Osorno, Río Bueno.

Chilicola (Oediscelis) lonco Toro \& Moldenke, 1979. An. Mus. Hist. Nat. 12: 111. Holótipo macho e alótipo na CTV. Parátipos no MHNS, AMNH, UCV e CTV.

Morfologia. Toro \& Moldenke, 1979: 171, figs 68 a 76 (caracteres do macho e da fêmea).

mantagua Toro \& Moldenke. CHILE, Valparaíso, Lilenes (localidade-tipo); Aconcagua, Papudo-Zapallar.

Chilicola (Heteroediscelis) mantagua Toro \& Moldenke, 1979. An. Mus. Hist. Nat. 12: 114. Holótipo macho e alótipo na CTV. Parátipos: 30 machos e 11 fêmeas depositados no MHNS, SEMK, MNH, UCV e CTV. 
Morfologia. Toro \& Moldenke, 1979: figs 77 a 85 (caracteres do macho e da fêmea). - Aravena \& Toro, 1985. Rev. Chil. Ent. 12: 179, figs 6, 13, 29 (ferrão e estruturas associadas).

mavida Toro \& Moldenke. CHILE, Coquimbo, Baños del Toro (localidade-tipo) Río Laguna.

Chilicola (Heteroediscelis) mavida Toro \& Moldenke, 1979. An. Mus. Hist.

Nat. 12: 122. Holótipo macho e alótipo na CTV. Sete parátipos depositados no MHNS, AMNH, SEMK, UCV e CTV.

Morfologia. Toro \& Moldenke, 1979: 173, figs 127 a 133 (caracteres do macho).

michelbacheri Toro \& Moldenke. CHILE, Cautín, Sierra de Nahuelbuta, W de Angol (localidade-tipo).

Chilicola (Idioprosopis) michelbacheri Toro \& Moldenke, 1979. An. Mus.

Hist. Nat. 12: 140. Holótipo macho depositado na CAS.

Morfologia. Toro \& Moldenke, 1979: 177, figs 236 a 242 (caracteres do macho).

neffi Toro \& Moldenke. CHILE, Antofagasta, Taltal (localidade-tipo).

Chilicola (Heteroediscelis) neffi Toro \& Moldenke, 1979. An. Mus. Hist.

Nat. 12: 118. Holótipo e alótipo na CTV. Parátipos no MHNS, AMNH,

SEMK, UCV e CTV.

Biologia. Toro \& Moldenke, 1979: 119 (coletados sobre Oxalis L., Cristaria Cav. e Balbisia peduncularis D. Don.).

Morfologia. Toro \& Moldenke, 1979: 173, figs 101 a 109 (caracteres do macho e da fêmea).

olmue Toro \& Moldenke. CHILE, Valparaíso, La Campana (localidade-tipo).

Chilicola (Heteroediscelis) olmue Toro \& Moldenke, 1979. An. Mus. Hist.

Nat. 12: 130. Holótipo macho na CTV. Parátipos: 5 machos depositados no MHNS, AMNH, SEMK e CTV.

Taxonomia. Michener, 1995: 337 (comentou que poderia pertencer ao subgênero Anoediscelis).

Morfologia. Toro \& Moldenke, 1979: 175, figs 175 a 183 (caracteres da fêmea e do macho).

orophila Toro \& Moldenke. CHILE, Aconcagua, Guardia Vieja (localidade-tipo).

Chilicola (Heteroediscelis) orophila Toro \& Moldenke, 1979. An. Mus.

Hist. Nat. 12: 129. Holótipo macho na CTV. Um parátipo macho no AMNH.

Taxonomia. Michener, 1995: 337 (comentou que poderia pertencer ao subgênero Anoediscelis).

Morfologia. Toro \& Moldenke, 1979: 175, figs 168 a 174 (caracteres do macho). 
solervicensi Toro \& Moldenke. CHILE, Coquimbo, Los Molles (localidade-tipo), Los Vilos.

Chilicola (Idioprosopis) solervicensi Toro \& Moldenke, 1979. An. Mus. Hist. Nat. 12: 141. Holótipo macho e alótipo na CTV. Cinco parátipos machos depositados no SEMK, AMNH, MHNS e CTV.

Morfologia. Toro \& Moldenke, 1979: 177, figs 243 a 251 (caracteres do macho e da fêmea).

travesia Toro \& Moldenke. ChILE, Atacama, Travesía (localidade-tipo), N de Caldera, Copiapó (Travesía).

Chilicola (Heteroediscelis) travesia Toro \& Moldenke, 1979. An. Mus. Hist. Nat. 12: 125. Holótipo e alótipo na CTV. Parátipos fêmeas e machos no SEMK, AMNH, MHNS, UCV e CTV.

Morfologia. Toro \& Moldenke, 1979: 173, figs 143 a 151 (caracteres do macho e da fêmea).

valparaiso Toro \& Moldenke. CHILE, Valparaíso, Peñuelas (localidade-tipo).

Chilicola (Heteroesdicelis)[sic] valparaiso Toro \& Moldenke, 1979. An.

Mus. Hist. Nat. 12: 117. Holótipo macho na CTV.

Morfologia. Toro \& Moldenke, 1979: 171, figs 93 a 100 (caracteres do macho).

vernalis (Philippi). CHILE (localidade-tipo de $O$. vernalis e de $P$. decolor), Atacama, Caldera, até Talca, Constitución, Concepción, Corral, Baños de Cauquenes.

Oediscelis vernalis Philippi, 1866. Stett. ent. Ztg. 27: 110. Síntipos no MHNS.

Prosopis decolor Sichel, 1867. Reise Novara, Zool. 2 (1): 143. Síntipo fêmea no NHMV, examinado por J.S. Moure.

Oediscelis verna [sic]; Ducke, 1910. Deutsch. ent. Ztschr.: 368.

Hylaeus decolor; Meade-Waldo, 1923. Gen. Insectorum 181: 31.

Chilicola (Oediscelis) vernalis; Toro \& Moldenke, 1979: 109.

Chilicola decolor; Toro \& Moldenke, 1979: 109; syn.: C. vernalis.

Taxonomia. Friese, 1906. Ztschr. Hym. Dipt. 6: 226 (redescrição, ocorrência). - Friese, 1908. Flora og Fauna 10: 9 (ocorrência). - Toro \& Moldenke, 1979: 109 (comentaram não ter examinado o tipo de Chilicola decolor citada frequentemente como sinônimo de $C$. vernalis).

Biologia. Toro \& Moldenke, 1979: 111 (sobre Baccharis paniculata DC.).

Morfologia. Toro \& Moldenke, 1979: 171, figs 59 a 67 (caracteres do macho e da fêmea). - Aravena \& Toro, 1985. Rev. Chil. Ent. 12: 178, figs 2, 12, 30 (ferrão e estruturas associadas). - Toro \& Magunacelaya, 1987. Acta Ent. Chilena 14: 15, figs 5 e 6 (músculos femurais).

vicugna Toro \& Moldenke. CHILE, Coquimbo, el Pangue (localidade-tipo).

Chilicola (Heteroediscelis) vicugna Toro \& Moldenke, 1979. An. Mus. Hist. Nat. 12: 120. Holótipo e alótipo na CTV. 
Biologia. Toro \& Moldenke, 1979: 121 (visitando flores de Pleurophora pusilla Hook. \& Arn.).

Morfologia. Toro \& Moldenke, 1979: figs 110 a 119 (caracteres do macho e da fêmea).

vina Toro \& Moldenke. CHILE, Valparaíso, Cuesta la Dormida (localidade-tipo); Valparaíso, El Salto.

Chilicola (Heteroediscelis) vina Toro \& Moldenke, 1979. An. Mus. Hist.

Nat. 12: 124. Holótipo macho e alótipo na CTV. Parátipos: seis machos e uma fêmea no MHNS, AMNH, SEMK, UCV e CTV.

Morfologia. Toro \& Moldenke, 1979: figs 134 a 142 (caracteres do macho e da fêmea).

\section{Chilicola (Prosopoides) Friese, 1908}

Prosopoides Friese, 1908. Flora og Fauna 10: 10. Espécie-tipo: Oediscelis paradoxus Friese, $1908=$ Oediscelis prosopoides Ducke, 1907. Monotípico.

Chilicola (Prosopoides); Michener, 1995: 340.

Taxonomia. Michener, 1995: 340 (explicou que o nome Prosopoides foi publicado casualmente por Friese e atribuído a Ducke, que não descreveu o gênero; comparou Prosopoides com Hylaeosoma e Pseudiscelis).

minima (Ducke). BRASIL, Ceará, Quixadá (localidade-tipo).

Oe.[sic] minima Ducke, 1908. Rev. d'Ent., Caen, 27: 63. Lectótipo fêmea no MPEG.

Chilicola (Prosopoides) minima; Michener, 1995: 340.

Taxonomia. Brooks \& Michener, 1999. Jour. Hym. Res. 8 (2); 137 (designaram o lectótipo fêmea que foi encontrado no SEMK e que seria retornado ao MPEG, confirmaram a espécie no subgênero Prosopoides).

Biologia. Ducke, 1908: 64 (em flores de Hyptis suaveolens (L.) Poit.).

prosopoides (Ducke). BRASIL, Minas Gerais, Barbacena (localidade-tipo de Oediscelis prosopoides e de Oediscelis paradoxus) $1.100-1.200 \mathrm{~m}$.

Oediscelis prosopoides Ducke, 1907. Ztschr. Hym. Dipt. 7: 363. Síntipos: uma fêmea coletada em 18.X.1905 e um macho coletado em 27.X.1905, no BMNH, examinados por J.S. Moure.

Oediscelis paradoxus Friese, 1908. Flora og Fauna 10: 9. Um síntipo fêmea no AMNH, coletado em 26.X.1905, examinado por J.S. Moure; syn. nov.

Chilicola (Prosopoides) prosopoides; Michener, 1995: 340.

Taxonomia. Ducke, 1907: 363 (comentou a aparência de $O$. prosopoides lembrando uma pequena Prosopis F., 1804 [= Hylaeus Fabricius, 1793] como também Nitela amazonica Ducke, 1903). - Friese, 1908: 9 (atribuiu Oediscelis paradoxus a Ducke). - Sandhouse, 1943. Proc. U. S. Natl. Mus. 92: 591(atribuiu Oediscelis paradoxus a Friese).

Biologia. Ducke, 1907: 363 (voando sobre flores de Cuphea $\mathrm{P}$. Br. de outubro a dezembro). 


\section{Chilicola (Pseudiscelis) Friese}

Pseudiscelis Friese, 1906. Ztschr. Hym. Dipt. 6: 228. Espécie-tipo: Pseudiscelis rostrata Friese, 1906. Monotípico.

Chilicola (Pseudiscelis); Michener, 1995: 341.

Taxonomia. Friese, 1908. Flora og Fauna 10: 10 (comparou com Oediscelis e Eriades [sic] [= Heriades Spinola, 1808]). - Michener, 1995: 341 (comentou haver somente uma espécie descrita de C. (Pseudiscelis)).

rostrata (Friese). ARgENTINA, Salta (localidade-tipo); La Rioja.

Pseudiscelis rostrata Friese, 1906. Ztschr. Hym. Dipt. 6: 228. Síntipo fêmea provavelmente no MNHU.

Oediscelis (Pseudiscelis) rostrata; Ducke, 1907. Ztschr. Hym. Dipt. 7: 363. Chilicola (Pseudiscelis) rostrata; Michener, 1995: 341.

Taxonomia. Michener, 1995: 341(comparou com Chilimelissa rozeni Toro \& Moldenke, 1979).

\section{Chilimelissa Toro \& Moldenke, 1979}

Chilimelissa Toro \& Moldenke, 1979. An. Mus. Hist. Nat. 12: 95. Espécie-tipo: Chilimelissa luisa Toro \& Moldenke. Designação original.

Taxonomia. Toro \& Moldenke, 1979: 97 (incluiram na chave); 149-150 (diagnose, espécies incluídas e chave para os machos e fêmeas). - Toro, 1997. Acta Ent. Chilena 21: 10-11 (chave para as espécies chilenas).

australis Toro \& Moldenke. ArgentinA, Santa Cruz (localidade-tipo), $8 \mathrm{~km} \mathrm{~S} \mathrm{de}$ Los Antiguos.

Chilimelissa australis Toro \& Moldenke, 1979. An. Mus. Hist. Nat. 12: 159. Holótipo macho na CTV.

Taxonomia. Toro \& Moldenke, 1979: 159 (compararam com C. farellones Toro \& Moldenke, 1979).

Morfologia. Toro \& Moldenke, 1979: 179, figs 354 a 360 (caracteres do macho).

brevimalaris Toro. CHILE, III Região, Cuesta Chañaral (localidade-tipo).

Chilimelissa brevimalaris Toro, 1981. An. Mus. Hist. Nat.14: 218. Holótipo macho, alótipo e dois parátipos machos na CTV.

Taxonomia. Toro, 1981: 220 (comentou a colocação da espécie na chave proposta por TORO \& MOLDENKE (1979), onde o macho sairia com C. irwini Toro \& Moldenke, 1979 e a fêmea com C. chillan Toro \& Moldenke, 1979).

chillan Toro \& Moldenke. CHILE, Nuble, Chillán (Termas) (localidade-tipo).

Chilimelissa chillan Toro \& Moldenke, 1979. An. Mus. Hist. Nat. 12: 160.

Holótipo fêmea na CTV. Parátipos: 21 fêmeas depositadas no AMNH,

MHNS, UCV e CTV.

Taxonomia. Toro, 1997. Acta Ent. Chilena 21: 9 (descreveu o macho).

Morfologia. Toro \& Moldenke, 1979: 177, figs 267 e 268 (caracteres da fêmea). 
chusmiza Toro. CHILE, Tarapacá, Chusmiza (localidade-tipo).

Chilimelissa chusmiza Toro, 1981. An. Mus. Hist. Nat.14: 220. Holótipo fêmea na CTV.

Taxonomia. Toro, 1981: 221(na chave proposta por TORO \& MOLDENKE (1979) sairia com C. farellones Toro \& Moldenke, 1979).

farellones Toro \& Moldenke. CHILE, Aconcagua, Río Blanco (localidade-tipo) $2.800 \mathrm{~m}$; Santiago, Farellones.

Chilimelissa farellones Toro \& Moldenke, 1979. An. Mus. Hist. Nat. 12:

155. Holótipo macho e alótipo na CTV. Parátipos: um macho e três fêmeas de Farellones e quatro fêmeas de Aconcagua, Río Blanco, depositados no ANMH, SEMK, MHNS e CTV.

Morfologia. Toro \& Moldenke, 1979: 179, figs 318 a 326 (caracteres do macho e da fêmea).

irwini Toro \& Moldenke. ArgentinA, Santa Cruz, $55 \mathrm{~km} \mathrm{~S} \mathrm{de} \mathrm{Perito} \mathrm{Moreno}$ (localidade-tipo).

Chilimelissa irwini Toro \& Moldenke, 1979. An. Mus. Hist. Nat. 12: 158. Holótipo macho na CTV.

Morfologia. Toro \& Moldenke, 1979: 179, figs 334 a 340 (caracteres do macho).

longipalpa Toro. CHILE, I Região, Chusmiza (localidade-tipo) $3.500 \mathrm{~m}$.

Chilimelissa longipalpa Toro, 1981. An. Mus. Hist. Nat.14: 217. Holótipo

fêmea e um parátipo fêmea na CTV.

Taxonomia. Toro, 1981: 218 (comentou que na chave de TORO \& MOLDENKE (1979) sairia com C. farellones e C. chillan).

luisa Toro \& Moldenke. CHILE, Antofagasta (localidade-tipo) Km 1687 Rodovia

Panamericana.

Chilimelissa luisa Toro \& Moldenke, 1979. An. Mus. Hist. Nat. 12: 150. Holótipo macho e alótipo na CTV. Parátipos: 34 machos e 25 fêmeas da mesma localidade depositados no SEMK, AMNH, MHNS, UCV e CTV.

Morfologia. Toro \& Moldenke, 1979: 177, figs 288 a 299 (caracteres do macho e da fêmea). - Aravena \& Toro, 1985. Rev. Chil. Ent. 12: 179, figs 8, 19, 26 (ferrão e estruturas associadas). - Toro \& Magunacelaya, 1987. Acta Ent. Chilena 14: 15, figs 15 a 18 (músculos femurais).

machi Toro. CHILE, VII Região, Laguna del Teno (localidade-tipo).

Chilimelissa machi Toro, 1997: 8. Acta Ent. Chilena 21: 8. Holótipo fêmea na CTV.

minuta Toro \& Moldenke. CHILE, Coquimbo, Vicuña (localidade-tipo).

Chilimelissa minuta Toro \& Moldenke, 1979. An. Mus. Hist. Nat. 12: 154. Holótipo fêmea na CTV. 
Taxonomia. Toro \& Moldenke, 1979: 154 (Compararam com C. luisa, C. mucar e $C$. rozeni, descritas no mesmo trabalho).

Morfologia. Toro \& Moldenke, 1979: 179, figs 348 e 349 (caracteres da fêmea).

mucar Toro \& Moldenke. CHILE, Cordillera de Antofagasta (localidade-tipo).

Chilimelissa mucar Toro \& Moldenke, 1979. An. Mus. Hist. Nat. 12: 152. Holótipo macho na CTV.

Taxonomia. Toro \& Moldenke, 1979: 152 (compararam com C. luisa).

Morfologia. Toro \& Moldenke, 1979: figs 327 a 333 (caracteres do macho).

nolanai Toro \& Moldenke. CHILE, Atacama, Caldera (localidade-tipo).

Chilimelissa nolanai Toro \& Moldenke, 1979. An. Mus. Hist. Nat. 12: 157. Holótipo fêmea na CTV.

Biologia. Toro \& Moldenke, 1979: 158 (coletado sobre flores de Nolana L.). Morfologia. Toro \& Moldenke, 1979: 179, figs 352 e 353 (caracteres da fêmea).

nortina Toro \& Moldenke. CHILE, Antofagasta (localidade-tipo).

Chilimelissa nortina Toro \& Moldenke, 1979. An. Mus. Hist. Nat. 12: 163. Holótipo macho provavelmente na CTV, os autores não citaram o depositário.

Morfologia. Toro \& Moldenke, 1979: 179, figs 341 a 345 (caracteres do macho).

obscura Toro. Chile, Coquimbo, Choros Bajos (localidade-tipo).

Chilimelissa obscura Toro, 1981. An. Mus. Hist. Nat. 14: 221. Holótipo fêmea na CTV.

Taxonomia. Toro, 1981: 222 (comparou com C. nolanai).

pedroi Toro \& Moldenke. CHILE, Antofagasta, Toconao (localidade-tipo).

Chilimelissa pedroi Toro \& Moldenke, 1979. An. Mus. Hist. Nat. 12: 161. Holótipo macho e alótipo na CTV. Seis parátipos fêmeas depositados no AMNH, SEMK, UCV e CTV.

Morfologia. Toro \& Moldenke, 1979: 179, figs 300 a 306 (caracteres do macho e da fêmea).

rozeni Toro \& Moldenke. CHILE, Atacama, Pueblo Hundido (localidade-tipo), Pan de Azúcar, Puquios.

Chilimelissa rozeni Toro \& Moldenke, 1979. An. Mus. Hist. Nat. 12: 153. Holótipo macho e alótipo na CTV. Parátipos: 5 fêmeas de Pan de Azúcar, 2 machos e 21 fêmeas de Puquios depositados no AMNH, SEMK, MHNS, UCV e CTV.

Morfologia. Toro \& Moldenke, 1979: 179, figs 307 a 317 (caracteres do macho e da fêmea). 
sielfeldi Toro \& Moldenke. CHILE, Atacama, Encanche (localidade-tipo).

Chilimelissa sielfeldi Toro \& Moldenke, 1979. An. Mus. Hist. Nat. 12: 161. Holótipo fêmea na CTV.

Morfologia. Toro \& Moldenke, 1979: 179, figs 350 e 351 (caracteres da fêmea).

xanthorhina Toro. CHILE, II Região, Taltal (localidade-tipo).

Chilimelissa xanthorhina Toro, 1997. Acta Ent. Chilena 21: 7. Holótipo macho e alótipo na CTV. Parátipos: 27 fêmeas e 6 machos depositados no MHNS, UCV, AMNH, SEMK e Universidad Metropolitana de Ciencias de la Educación, CHILE.

Biologia. Toro, 1997: 8 (associada exclusivamente com Philippiamra celosioides Kuntze).

\section{Geodiscelis Michener \& Rozen, 1999}

Geodiscelis Michener \& Rozen, 1999. Amer. Mus. Novitates 3281: 2. Espécie-tipo: Geodiscelis megacephala Michener \& Rozen, 1999. Designação original.

megacephala Michener \& Rozen. Argentina, San Juan, 16 km W de Media Agua (localidade-tipo).

Geodiscelis megacephala Michener \& Rozen, 1999: 2. Holótipo fêmea e 33 parátipos no AMNH. Um macho e uma fêmea parátipos no MACN, SEMK e CTV

Biologia. Michener \& Rozen, 1999: 7 (observaram muitos indivíduos voando sobre área arenosa e visitando flores de Heliotropium curassavicum L.; descreveram os ninhos, tendo examinado 7 ou 8 , em dois deles a galeria com ramificação e uma única célula no fim, as células com revestimento tipo celofane e fechadas com este material).

\section{Xenochilicola Toro \& Moldenke, 1979}

Xenochilicola Toro \& Moldenke, 1979. An. Mus. Hist. Nat. 12: 145. Espécie-tipo: Xenochilicola mamigna Toro \& Moldenke, 1979.

Taxonomia. Toro \& Moldenke, 1979: 145 (chave para as espécies).

diminuta Toro \& Moldenke. CHILE, Santiago, Qda. [Quebrada] La Plata (localidade-tipo).

Xenochilicola diminuta Toro \& Moldenke, 1979. An. Mus. Hist. Nat. 12:

147. Holótipo macho e três parátipos machos na CTV.

Morfologia. Toro \& Moldenke, 1979: 177, figs 278 a 285 (caracteres dos machos).

fulva Toro \& Moldenke. CHILE, Antofagasta, Taltal (localidade-tipo).

Xenochilicola fulva Toro \& Moldenke, 1979. An. Mus. Hist. Nat. 12: 148. Holótipo fêmea na CTV. Nove parátipos fêmeas, depositados no AMNH, SEMK, MHNS, UCV e CTV. 
Biologia. Toro \& Moldenke, 1979: 148 (coletados sobre Cristaria Cav.). Morfologia. Toro \& Moldenke, 1979: 177, figs 286 e 287 (caracteres da fêmea).

mamigna Toro \& Moldenke. CHILE, Tarapacá, Mamiña (localidade-tipo); Antofagasta, Chiu-Chiu.

Xenochilicola mamigna Toro \& Moldenke, 1979. An. Mus. Hist. Nat. 12: 145. Holótipo macho e alótipo na CTV. Parátipos: 10 machos e 70 fêmeas depositados no SEMK, AMNH, MHNS, UCV e CTV.

Biologia. Toro \& Moldenke, 1979: 147 (coletados sobre Baccharis petiolata DC. e Baccharis juncea Desf.).

Morfologia. Toro \& Moldenke, 1979: 177, figs 269 a 277 (caracteres do macho e da fêmea). - Aravena \& Toro, 1985. Rev. Chil. Ent. 12: 183, figs 10, 16, 25 (ferrão e estruturas associadas). - Toro \& Magunacelaya, 1987. Acta Ent. Chilena 14: 15, figs 19 a 22 (músculos femurais).

\section{Xeromelissa Cockerell, 1926}

Xeromelissa Cockerell, 1926. Ann. Mag. Nat. Hist. (9) 17: 221. Espécie-tipo: Xeromelissa wilmattae Cockerell, 1926: 222. Monotípico.

Taxonomia. Cockerell, 1926: 221-222 (descreveu o gênero tendo em mãos uma fêmea; comentou ser próximo de Euryglossa Smith, 1853, da Austrália). - Michener, 1944: 240 (comentou que Xeromelissa provavelmente pertenceria à subfamília Chilicolinae). - Toro \& Moldenke, 1979: 163-164 (redescreveram o gênero).

wilmattae Cockerell. PERU, Tingo [Maria] (localidade-tipo) Arequipa. CHILE, Tarapacá, Chusmiza.

Xeromelissa wilmattae Cockerell, 1926. Ann. Mag. Nat. Hist. (9) 17: 222. Holótipo fêmea depositado no AMNH.

Taxonomia. Toro \& Moldenke, 1979: 164 (redescreveram a fêmea e citaram ocorrência no Chile). - Toro, 1981. An. Mus. Hist. Nat. 14: 222 (descreveu o macho). - Toro \& Magunacelaya, 1987. Acta Ent. Chilena 14: 15, figs 23 a 26 (músculos femurais).

Biologia. Cockerell, 1926: 223 (em flores de Cristaria multifida Cav.).

Morfologia. Toro \& Moldenke, 1979: 179, figs 346 e 347 (caracteres da fêmea). - Aravena \& Toro, 1985. Rev. Chil. Ent. 12: 179, figs 9, 18, 24 (ferrão e estruturas associadas).

AGRADECIMENTOS. Ao Prof. Ms.C. Olavo Araujo Guimarães (Departamento de Botânica, UFPR) pela colaboração na nomenclatura das plantas, ao Prof. Dr. Albino M. Sakakibara e Prof. Dr. Gabriel A. R. Melo (Departamento de Zoologia, UFPR) pelos esclarecimentos sobre a taxonomia e empréstimo de bibliografia. 


\section{REFERÊNCIAS BIBLIOGRÁFICAS}

Cockerell, T.D.A. 1926. XXVIII. Descriptions and Records of Bees. CVIII. Ann. Mag. Nat. Hist. 17 (9): 214-226.

MiCHENER, C.D. 1944. Comparative external morphology, phylogeny, and a classification of the bees (Hymenoptera). Bull. Amer. Mus. Nat. Hist. 82 (6): 151-326.

. 1995. A Classification of the Bees of the Subfamily Xeromelissinae (Hymenoptera: Colletidae). Jour. Kansas Ent. Soc. 68 (3): 332-345.

2000. The Bees of the World. London, The John Hopkins Univ. Press, I-XIV + 913p.

Michener, C.D. \& J.G. Rozen JR. 1999. A New Ground-Nesting Genus of Xeromelissine Bees from Argentina and the Tribal Classification of the Subfamily (Hymenoptera: Colletidae). Amer. Mus. Novit. 3281: 1-10.

Moure, J.S. 1946. Notas sobre abelhas da sub família Chilicolinae (Hym. - Apoidea). Livro de homenagem a R.F. d'Almeida 27, p. 243-250.

Toro, H. \& C.D. Michener. 1975. The subfamily Xeromelissinae and its occurrence in Mexico (Hymenoptera: Colletidae). Jour. Kansas Ent. Soc. 48 (3): 351-357.

Toro, H. \& A. MoldenKE, 1979. Revision de los Xeromelissinae chilenos (Hymenoptera - Colletidae).

An. Mus. Hist. Nat. 12: 95-182.

Recebido em 19.X.2001; aceito em 25.II.2002.

\section{ÍNDICE DOS TAXA DE XEROMELISSINAE}

aequatoriensis Benoist, Chilicola 2

aisenensis Toro \& Moldenke, Chilicola (Chilicola) 1.8

albida $($ Friese $)=$ Chilicola minor .6

andina Toro \& Moldenke, Chilicola

(Chilioediscelis) . .8

araucana Toro \& Moldenke, Chilicola

(Chilioediscelis)

ashmeadi (Crawford), Chilicola

(Anoediscelis) 3

australis Toro \& Moldenke, Chilimelissa ........17

brevimalaris Toro, Chilimelissa. 17

chalcidiformis (Meade-Waldo), Chilicola (Oediscelis) 11

Chilicola Spinola ...2

Chilicola (Anoediscelis) Toro \& Moldenke ........ 3

Chilicola (Chilicola) Spinola 7

Chilicola (Chilioediscelis) Toro \& Moldenke .8

Chilicola (Heteroediscelis) Toro \& Moldenke $=$ Chilicola $($ Oediscelis $) \ldots .11$

Chilicola (Heteroesdiscelis) [sic] Toro \& Moldenke $=$ Chilicola (Oediscelis) ............11 Chilicola (Hylaeosoma) Ashmead ....................... 9 Chilicola (Oediscelis) Philippi ............................ 11 Chilicola (Prosopoides) Friese .......................... 16 Chilicola (Pseudiscelis) Friese......................... 17 Chilicola (Stenoediscelis) Toro \& Moldenke = Chilicola (Anoediscelis)......................... 3

Chilimelissa Toro \& Moldenke........................... 17

chillan Toro \& Moldenke, Chilimelissa ........... 17

chusmiza Toro, Chilimelissa .............................. 18

colliguay Toro \& Moldenke, Chilicola (Chilicola) .............................................. 8

colliquay $[\mathbf{s i c}]=C$. $($ Chilicola $)$ colliguay .......... 8 colliquey $[$ sic $]=C$. $($ Chilicola $)$ colliguay ............ 8 costaricensis (Friese) $=C$. (Anoediscelis) ashmeadi ............................................... 4

cupheae (Schrottky), Chilicola ............................. 3 dalmeidai (Moure), Chilicola (Oediscelis) ...... 12 decolor $($ Sichel $)=$ Chilicola vernalis ................ 15 deserticola Toro \& Moldenke, Chilicola (Oediscelis). 12 
diaguita Toro \& Moldenke, Chilicola

(Oediscelis) ...12

diminuta Toro \& Moldenke, Xenochilicola......20

erithropoda Toro \& Moldenke, Chilicola

(Oediscelis)

farellones Toro \& Moldenke, Chilimelissa .......18

friesei (Ducke), Chilicola (Oediscelis) ...............12

friesei Herbst nom. praeoc. $=$ Chilicola

plebeia

friesella nom. nov. para Chilicola friesei

Herbst, 1920 .7

fulva Toro \& Moldenke, Xenochilicola ............20

Geodiscelis Michener \& Rozen .20

gracilis Michener \& Poinar, Chilicola

(Hylaeosoma) .9

griswoldi Michener, Chilicola (Hylaeosoma) ....9

gutierrezi Moure, Chilicola (Oediscelis)..........13

hahni Herbst, Chilicola (Oediscelis) .................13

herbsti (Friese), Chilicola (Anoediscelis) ............4

howardiella $($ Cockerell $)=$

C. (Anoediscelis) ashmeadi .4

huberi (Ducke), Chilicola (Hylaeosoma) ..........10

Hylaeosoma Ashmead = Chilicola

(Hylaeosoma)

Hyloeosoma $[\mathrm{sic}]$ Ashmead $=$ Chilicola

(Hylaeosoma) .9

Idioprosopis Meade-Waldo $=$ Chilicola

(Oediscelis) 11

inermis (Friese), Chilicola (Anoediscelis) ...........5

irwini Toro \& Moldenke, Chilimelissa .............18

lonco Toro \& Moldenke, Chilicola

(Oediscelis)

longiceps (Ashmead), Chilicola

(Hylaeosoma) .10

longipalpa Toro, Chilimelissa ...........................18

longitarsa Spinola, Chilicola ................................... 3

luisa Toro \& Moldenke, Chilimelissa ...............18

machi Toro, Chilimelissa ...................................18

mailen Toro \& Moldenke, Chilicola

(Anoediscelis) ...........................................6

mamigna Toro \& Moldenke, Xenochilicola .....21

mantagua Toro \& Moldenke, Chilicola

(Oediscelis)

mavida Toro \& Moldenke, Chilicola

(Oediscelis) megacephala Michener \& Rozen,

Geodiscelis

megalostigma (Ducke), Chilicola

(Hylaeosoma).

mexicana Toro \& Moldenke, Chilicola

(Hylaeosoma)

michelbacheri Toro \& Moldenke, Chilicola

(Oediscelis) ..................................... 14

minima (Ducke), Chilicola (Prosopoides) ....... 16

minor (Philippi), Chilicola (Anoediscelis) ..........6

minuta Toro \& Moldenke, Chilimelissa ............ 18

mucar Toro \& Moldenke, Chilimelissa ........... 19

neffi Toro \& Moldenke, Chilicola

(Oediscelis)

nolanai Toro \& Moldenke, Chilimelissa .......... 19

nortina Toro \& Moldenke, Chilimelissa .......... 19

obscura Toro, Chilimelissa ................................19

Oediscelis Philippi $=$ Chilicola $($ Oediscelis $)$.....11

Oediscelisca Moure $=$ Chilicola

(Oediscelis)

olmue Toro \& Moldenke, Chilicola

(Oediscelis)

orophila Toro \& Moldenke, Chilicola

(Oediscelis) 14

pangue Toro \& Moldenke, Chilicola

(Chilicola)

paradoxus $($ Friese $)=$ Chilicola

(Prosopoides) prosopoides 16

patagonica Toro \& Moldenke, Chilicola

(Chilioediscelis)

pedroi Toro \& Moldenke, Chilimelissa ...........19

plebeia Spinola, Chilicola (Anoediscelis) .......... 7

plebeja $[$ sic $]=C$. $($ Anoediscelis $)$ plebeia ............ 7

polita Michener, Chilicola (Hylaeosoma)........11

Prosopoides Friese $=$ Chilicola $($ Prosopoides) 16 prosopoides (Ducke), Chilicola (Prosopoides) 16 Pseudiscelis Friese $=$ Chilicola $($ Pseudiscelis) 17 quitensis Benoist, Chilicola.

rostrata (Friese), Chilicola (Pseudiscelis) ........17

rozeni Toro \& Moldenke, Chilimelissa ............. 19

rubriventris Spinola, Chilicola (Chilicola) ........ 8

sielfeldi Toro \& Moldenke, Chilimelissa ..........20

solervicensi Toro \& Moldenke, Chilicola

(Oediscelis) 
stenocephala Brooks \& Michener,

Chilicola (Hylaeosoma)

styliventris (Friese), Chilicola

(Anoediscelis).

travesia Toro \& Moldenke, Chilicola

(Oediscelis)

valparaiso Toro \& Moldenke, Chilicola

(Oediscelis) 15

verna $[\mathbf{s i c}]=C$. (Oediscelis) vernalis

vernalis (Philippi), Chilicola (Oediscelis).........15

vicugna Toro \& Moldenke, Chilicola

(Oediscelis)

vina Toro \& Moldenke, Chilicola

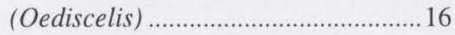

xantorhina Toro, Chilimelissa......

Xenochilicola Toro \& Moldenke. .20

Xeromelissa Cockerell. .21

wilmattae Cockerell, Xeromelissa 21

yali Toro \& Moldenke, Chilicola

(Anoediscelis). . .7

\section{ÍNDICE DAS PLANTAS}

Achillaea [sic] millefolium

Achillea millefolium L. [Asteraceae]..........5, 6, 7

Adesmia melanthes Phil. [Fabaceae]................... 8

Anthemis cotula Blanco. [Compositae] ......5, 6, 7

Antirrhinum majus L. [Scrophulariaceae]...........5

Aquilegia vulgaris L. [Ranunculaceae] ............... 6

Aquilejia [sic] vulgaris.

Armeria maritima (Miller) Willd.

[Plumbaginaceae]..

Azara celastrina D. Don. [Flacourtiaceae] ....5, 7 Azara integrifolia Ruiz \& Pav.

[Flacourtiaceae].

Baccharis confertifofolia Colla

[Asteraceae]

Baccharis juncea Desf. [Asteraceae]

Baccharis paniculata DC. [Asteraceae]........... 15

Baccharis petiolata DC. [Asteraceae].

.21

Baccharis rosmarinifolia Hook. \& Arn.

[Asteraceae] $5,6,7$

Baccheris [sic] confertifolia ....6

Baccheris [sic] rosmarinifolia $5,6,7$

Balbisia peduncularis D. Don.

[Geraniaceae].
Bambus [Poaceae] 6

bambu da India 6

Bambusa Schref. [Poaceae] ............................... 6

Bidens pilosa L. [Asteraceae] ............................ 4

Borreria verticillata G.F.W. Mey.

[Rubiaceae]

Brassica campestris L. [Brassicaceae] .............. 5

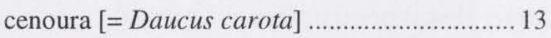

Cheiranthus cheiri L. [Brassicaceae].................. 6

Colletia ferox Gill. \& Hook. [Rhamnaceae] ...... 6

Compostas [Asteraceae] .................................... 6

Cristaria Cav. [Malvaceae] 14,21

Cristaria intermedia Gay [Malvaceae].......... 5, 6

Cristaria multifida Cav. [Malvaceae] ............... 21

Cuphea P. Br. [Lythraceae] ...................... 11, 16

Cuphea mesostemon Koehne [Lythraceae]........ 3

Daucus carota L. [Apiaceae] ............................. 13

Drymaria Willd ex Schult. [Caryophyllacea].. 13

Echinocactus ceratistes Otto ex Pfeiff.

[Cactaceae] 6

Echinocactus ceratites [sic] 6

Eryngium paniculatum Cav. \& Dombey ex

Delar. [Apiaceae]

Erythrina poeppigiana (Skeels) [Fabaceae] ...... 4

Escallonia pulverulenta Pers.

[Saxifragaceae]

Escalonia [sic] pulverulenta …............................ 6

Galinsoga Ruiz \& Pav. [Asteraceae] .................. 4

Gymnophyton polycephalum $\mathrm{Clos}$

[Apiaceae]

Gymnophytum [sic] polycephalum .

Helianthus tuberosus L. [Asteraceae] .................. 6

Heliotropium curassavicum $\mathrm{L}$.

(Boraginaceae) 20

Heliotropium stenophilum [sic] .....

Heliotropium stenophyllum Hook. \&

Arn. [Boraginaceae]

Hypochaeris [sic] lessingii.

Hypochoeris lessingii Reiche [Asteraceae] ....... 5

Hyptis suaveolens (L.) Poit. [Lamiaceae] ......... 16

Jaegeria hirta Less. [Asteraceae] ....................... 4

Larrea divaricata Cav. [Zygophyllaceae] ......... 5

Leuceria eriochlaena Remy [Compositae] ........ 5

Leuceria eriochloena $[\mathrm{sic}]$............................... 5 
Lippia citriodora H.B.K. [Verbenaceae].............7

Loasa tricolor Lindl. Weinm. [Loasaceae] ....6, 7

Lobelia polyphylla Fr. Meigen

[Campanulaceae]. ...7

Lobelia salicifolia Sweet [Campanulaceae] ........5

Lychnis L. [Caryophyllaceae]...............................

Matthiola annua Sweet [Brassicaceae] ...............5

Melampodium divaricatum (Rich.) DC. [Asteraceae] ....4

Nolana L. [Convolvulaceae] …...........................19

Oxalis L. [Oxalidaceae] . .14

Phacelia circinata Jacq. F. [Hydrophyllaceae] $.5,6,13$

Philippiamra celosioides Kuntze

[Portulacaceae]

Phrygilanthus aphyllus Eichl.

[Loranthaceae]. .7

Pleurophora pusilla Hook. \& Arn.

[Lythraceae] .16

Podanthus mitiqui Lindl. [Asteraceae]................. 5

Polymnia maculata Cav. [Asteracae] ...................4

Raphanus radiola DC. [Brassicaceae] ................. 3

Rubus L. [Rosaceae] .............................................

Rubus fruticosus L. [Rosaceae]............................ 3

Ruellia L. [Acanthaceae] ....................................10

Salvia L. [Lamiaceae]............................................

Salvia splendens Ker Gawl. [Lamiaceae]........5, 6

Santolina chamaecyparissus L.

[Asteraceae] .6

sarça [= Rubus fruticosus $]$

Saracha procumbens (Cav.) Ruiz \& Pavón [Solanaceae] .4

Sedum L. [Crassulaceae] $.5,7$

Senecio adenotrichius DC. [Asteraceae]............7

Senecio rutaceus Phil. [Asteraceae] .....................7

Solanaceae .............................................................. 4

Solanum nigrum L. [Solanaceae] ........................ 4
Solanum umbellatum Mill. [Solanaceae] ............ 4

Sonalum wendlandii Hook. [Solanaceae] ...........4 4

Stachys albicaulis Lindl. [Lamiaceae] ................ 5

Tanacetum parthenifolium Sch. Bip.

[Asteraceae] 5,6

Taraxacum dens-leonis Desf.

$[=$ Taraxacum officinale $]$..................... 5

Taraxacum officinale Weber [Asteraceae]..........5

Tecoma Juss. [Bignoniaceae] …………………..... 5

Tripogandra amplexicaulis (Klotz. ex B Clarke) Woodson [Commelinaceae]....9

\section{OUTROS INSETOS}

Besouros [Coleoptera] 6

Calliopsis Smith, 1853 [Hymenoptera,

Andrenidae] .9

Ceratina Latreille, 1802[Hymenoptera,

Anthophoridae] .7

Dasypoda Latreille, 1802 [Hymenoptera, Melittidae] 9

Eriades [sic] [= Heriades Spinola, 1808]

[Hymenoptera, Megachilidae]

Euryglossa Smith, 1853 [Hymenoptera,

Colletidae]

Hylaeus Fabricius, 1793 [Hymenoptera,

Colletidae]

Manuelia Vachal, 1905 [Hymenoptera,

Anthophoridae] .7

Mosquitos [Diptera] ..... 6

Nitela amazonica Ducke, 1903 [Hymenoptera,

Crabronidae, Crabroninae]................... 16

Prosopis Fabricius, 1804 [= Hylaeus Fabricius, 1793] [Hymenoptera, Colletidae] ...... 16

Scrapteroides Gribodo, 1894 [Hymenoptera, Andrenidae]. .7

Stigmus (Gonostigmus) temporalis [= Stigmus temporalis Kohl, 1892] [Hymenopterả, Crabronidae, Pemphredoninae] 4 